\title{
MCTS1 promotes invasion and metastasis of oral cancer by modifying the EMT process
}

\author{
Zhexun Huang ${ }^{1}$, Qiao Su${ }^{2}$, Wuguo $\mathrm{Li}^{2}$, Hui Ren ${ }^{1}$, Huiqiang Huang ${ }^{3,4}$, Anxun Wang ${ }^{1}$ \\ ${ }^{1}$ Department of Oral and Maxillofacial Surgery, the First Affiliated Hospital, Sun Yat-sen University, Guangzhou, China; ${ }^{2}$ Animal Experiment \\ Center, the First Affiliated Hospital, Sun Yat-sen University, Guangzhou, China; ${ }^{3}$ Department of Medical Oncology, Sun Yat-sen University Cancer \\ Center, Guangzhou, China; ${ }^{4}$ State Key Laboratory of Oncology in South China, Guangzhou, China; Collaborative Innovation Center for Cancer \\ Medicine, Guangzhou, China \\ Contributions: (I) Conception and design: Z Huang, A Wang; (II) Administrative support: W Li; (III) Provision of study materials or patients: H \\ Ren, H Huang; (IV) Collection and assembly of data: Z Huang; (V) Data analysis and interpretation: Z Huang, Q Su; (VI) Manuscript writing: All \\ authors; (VII) Final approval of manuscript: All authors. \\ Correspondence to: Anxun Wang. Department of Oral and Maxillofacial Surgery, the First Affiliated Hospital, Sun Yat-Sen University, Guangzhou \\ 510080, China. Email: wanganx@mail.sysu.edu.cn.
}

\begin{abstract}
Background: The oncogene, malignant T-cell-amplified sequence 1 (MCTS1), has been found to be highly expressed in a variety of cancer cell lines. It has been shown to be involved in cell cycle progression and to confer a growth advantage for lymphomas and breast cancer. Nevertheless, the role of MCTS1 in contributing to the development of oral cancer remains elusive.

Methods: We analyzed the gene expression profiles of MCTS1 in normal oral keratinocytes and cancerous cells. Cellular proliferation, invasion, and migration experiments were performed to detect the effect of MCTS1 on the biological evolution of oral cancer. The in vitro results were verified by the in vivo lymphatic metastasis test. The underlying mechanism of MCTS1 in promoting oral cancer invasion and metastasis correlated with the epithelial-mesenchymal transition (EMT) process as revealed by western blotting.

Results: The results showed that MCTS1 was aberrantly expressed in oral cancer cells. MCTS1 overexpression significantly promoted tumor cell growth, proliferation, migration, and invasion. MCTS1mediated lymphatic metastasis was verified in vivo using an intraplantar tumor model. Biomarkers associated with EMT progression were positively or negatively regulated upon knockdown or overexpression of MCTS1, respectively.

Conclusions: Higher MCTS1 expression in oral cancer may be connected with an unfavorable prognosis due to involvement of MCTS1. MCTS1 potentiates the growth and proliferation of oral cancer cells and subsequent metastasis by regulating cell cycle and modifying the EMT process.
\end{abstract}

Keywords: Oral cancer; oncogene; malignant T-cell-amplified sequence 1 (MCTS1); metastasis; invasion

Submitted May 01, 2021. Accepted for publication May 28, 2021.

doi: 10.21037/atm-21-2361

View this article at: http://dx.doi.org/10.21037/atm-21-2361

\section{Introduction}

Oral cancer is a kind of cancer that develops in the tissues of the mouth or throat, primarily being generated in the squamous cell lining in the mouth, tongue, and lips. More than 657,000 new cases of oral cancer are diagnosed annually according to World Health Organization (WHO).
Research has demonstrated the critical role of oncogene malignant T-cell-amplified sequence 1 (MCTS1) in the invasion and metastasis of cancers (1). The neoplastic transformation of oncogenesis can be induced through the mutation of specific genes. This commonly involves the gain of an oncogene function or loss of a tumor-suppressor gene function. The stringent control of critical cell 
biochemical processes manages normal cellular behaviors, like proliferation, cycle, and apoptosis (2). Oncogenes dominate the mechanisms of cellular transformation via signal transduction that releases extracellular messages to the nucleus, resulting in variational cellular transcriptional patterns (3). Genetic modifications that break the balance between oncogenes and tumor suppressors can disturb the cellular signaling pathways, causing initiation of neoplastic properties (4).

MCTS1 plays multiple roles in the carcinogenesis and development of malignant tumors (1). MCTS1 can affect the cell cycle by shortening the G1/S transition time, which is achieved by disequilibrating the G1 cyclins between activators and inhibitors (5). An increased expression of MCTS1 following DNA damage has been identified $(6,7)$. MCTS1 allows for the circumvention of the G1/S checkpoint and directs cells into the $\mathrm{S}$ phase following DNA damage. The elevated MCTS1 protein level following DNA damage is attributed to posttranslational modification, where phosphorylation of MCTS-1 by $\mathrm{p} 44 / \mathrm{p} 42$ MAPK is critical for stabilization of protein the ability to promote cell proliferation (8). MCTS1 also plays a role as a translation enhancer. It recruits density regulated re-initiation and release factor (DENR) and binds to the cap complex of the 5'-terminus of messenger RNAs (mRNAs), thus altering the mRNA translation profile (9). In fact, translational control has been proven to be an important step in regulating the gene expression and subsequent supervision of cell growth, differentiation, and necrocytosis. Additionally, MCTS1 exhibits potential effects on angiogenesis and apoptosis. It has been demonstrated that MCTS1 contributes to in vivo tumorigenicity of MCF7 cells by promoting angiogenesis and inhibiting apoptosis (10). Overall, MCTS1 is a unique oncogene that can act on a subpopulation of cancer cells through an array of mechanisms to deregulate the cell cycle, abnormally activate kinases, escalate DNA damage, and enable other oncogenes, thus promoting tumor invasion and metastasis.

Oral cancer has become one of the most common squamous cell carcinomas of the head and neck (11). Its diagnosis at a relatively advanced disease stage contributes to its poor prognosis in patients. Thus, identifying new biomarkers that can help to ameliorate the curative effect by means of timely intervention is urgently needed. While the relevance of MCTS1 to B-cell lymphomas (12) and breast cancer $(13,14)$ has been confirmed, it would be of further interest to delineate the interrelation between MCTS1 and oral cancer and confirm whether MCTS1 is able to promote invasion and metastasis of squamous cell carcinomas.

Herein, we analyzed MCTS1 expression in normal human oral keratinocytes (HOK) and various oral cancer cell lines (SCC9, SCC15, SCC25, and Cal27) and surveyed the association between MCTS1 expression and the progression of oral cancer. We constructed a stable SCC9 cell line with an MCTS1-knockdown and MCTS1-overexpressed SCC25 cell line by lentiviral transduction in order to investigate the effects of MCTS1 on cell proliferation, migration, and metastasis, as well as on mesenchymal transition (EMT). The present study suggests that high expression of MCTS1 promotes invasion and metastasis of oral cancer cells and modifies the EMT process, which in turn contributes to cell proliferation and migration.

We present the following article in accordance with the ARRIVE reporting checklist (available at http://dx.doi. org/10.21037/atm-21-2361).

\section{Methods}

\section{Cell lines and culture}

HOK and oral cancer cell lines (SCC9, SCC15, SCC25 and Ca127) were obtained from the American Type Culture Collection (ATCC) and cultured in Dulbecco's Modified Eagle Medium (DMEM)/F12 medium supplemented with $10 \%$ fetal bovine serum (FBS, Gibco, USA) and $1 \%$ penicillin-streptomycin (Gibco, USA). Virus-packaging cells (MCTS1-overexpressed SCC9 and MCTS-knockdown SCC25) were cultured in the same medium in addition to $4 \mu \mathrm{g} / \mathrm{mL}$ puromycin. Cells were cultured at $37{ }^{\circ} \mathrm{C}$ in a humidified $5 \% \mathrm{CO}_{2}$ atmosphere. All cell lines were negative for mycoplasma. No cell lines used in this study were found in the database of commonly misidentified cell lines that are maintained by International Cell Line Authentication Committee (ICLAC) and NCBI biosample.

\section{Tissue specimens and microarray construction}

Specimens of tumor tissue excised from the patients with head and neck cancer were collected at the Department of Oral and Maxillofacial Surgery, First Affiliated Hospital of Sun Yat-sen University from November 2016 to April 2019. The study was conducted in accordance with the Declaration of Helsinki (as revised in 2013). All samples were obtained with informed consent and their use was approved by the Ethics Committee of the First Affiliated 
Hospital of Sun Yat-sen University (No. 2016074). Formalin-fixed paraffin embedded tissue samples were picked up from the library of specimens. Representative areas were selected for the construction of tissue microarrays (TMAs) specifically designed to analyze the target gene of MCTS1. Two different sets of TMAs were prepared, including tissues with lymph node metastasis and nonmetastasis. Each sample was scored according to the proportion of positively stained cells (proportion score, $0-4$ ) and the staining intensity (intensity score, $0-3$ ). The staining index was calculated by multiplying the proportion score and the intensity score.

\section{DNA constructs and gene transfection}

For overexpression of MCTS1, recombinant lentivirusexpressing MCTS1(LPP-Lv105-MCTS1, GeneCopoeia, USA) and empty plasmid cloning DNA control vector were adopted to transfect the SCC9 cell line. For knockdown of MCTS1, predesigned short hairpin RNAs (siRNAs; HsH045142, GeneCopoeia, USA) were constructed into pReceiver-Lv105 for lentivirus mediated transfection. Stable transfection was performed in the SCC25 cell line to knock down the MCTS1 gene, and puromycin-resistant clones were subsequently propagated. Oligonucleotide and lentiviral transfection were performed according to previously published procedures (15). Western blotting and real-time polymerase chain (RT-PCR) were used to detect the expression of the target genes.

\section{Gene expression array and real-time PCR validation}

RNA was extracted from cell lines using Trizol (T9424, Sigma-Aldrich, USA), and complement DNA (cDNA) was synthesized with the PrimeScript RT-PCR Kit (RR014B, Takara, Japan). Gene expression was analyzed by TB Green Fast qPCR Mix (RR430S, Takara) on a CFX384 Real-Time System (Bio-Rad, USA). Gene expression was validated by RT-PCR using the nucleotide sequences of 5'-TGGCTTAATCAAATCATGCCTA-3' and 5'-GTACAAATTTGATGGCTCCTTT-3' as the forward primer and reverse primer, respectively. Reactions were performed in triplicate.

\section{Immunoblotting}

Cells were gently scraped in lysis buffer containing $25 \mathrm{mM}$ of Tris (pH 7.6), $15 \mathrm{mM}$ of $\mathrm{NaCl}, 1 \% \mathrm{NP}-40$, and a protease inhibitor cocktail. Protein concentration was quantified by the bicinchoninic acid (BCA) method. Protein (20 mg) was loaded onto $5-15 \%$ polyacrylamide gel and subjected to immunoblot analysis with the target antibodies. Antibodies against $\beta$-actin were adopted to detect the respective protein levels. All primary antibodies were diluted as per 1:1,000 in blocking solution [5\% milk, $0.1 \%$ Tween 20 in tris-buffered saline (TBS)]. The immunoblots were blocked for 1 hour at room temperature in $5 \%$ nonfat milk (TBS, $0.1 \%$ Tween 20) followed by an overnight incubation at $4{ }^{\circ} \mathrm{C}$ in corresponding diluted solutions containing the primary antibody. Membranes were then flushed 3 times using TBS containing $0.1 \%$ Tween 20 and further incubated with the secondary antibody for another 1 hour at room temperature. All secondary antibodies were diluted in a 1:2,000 concentration in the blocking solution. All blots were developed using the Pierce ECL Western blot substrate (Thermo Fisher Scientific, USA). MCTS1-associated protein and important EMT markers like E-cadherin, $\mathrm{N}$-cadherin, Vimentin, $\beta$-catenin, Snail, and Slug were checked by the established western blotting method using cell samples. Each experiment was repeated a minimum of 3 times with 3 independently prepared protein samples.

\section{Clonogenic assay and MTT testing}

After transfection, 800 cells were dropped in $6-\mathrm{cm}$ culture dishes for 14 days. Colonies were fixed with $100 \%$ precooling methanol, stained with $5 \%$ crystal violet, and counted. To investigate the proliferation of different cell lines, aliquots of cells were seeded in 96-well plate for 24 hours, and $3 \times 10^{3}$ cells that were seeded into 96-well plates were used for MTT assay. At the predetermined time (0, 1, 2 and 4 d), MTT (M5655, Sigma-Aldrich) was added to each well to a final concentration of $0.5 \mathrm{mg} / \mathrm{mL}$ followed by incubation for 4 hours at $37^{\circ} \mathrm{C}$. Then, the medium was removed and $100 \mu \mathrm{L}$ of dimethyl sulfoxide (DMSO) was introduced into each well. The absorbance at $570 \mathrm{~nm}$ was measured using a microplate reader.

\section{Wound healing migration assay}

MCTS1-overexpressed SCC9 and MCTS1-knockdown SCC25 cells in exponential growth were seeded into 6-well tissue culture plates and cultured in non-FBS medium at the density of $1 \times 10^{6}$ cells $/ \mathrm{mL}$. When a confluent cell monolayer formed, the monolayer was scratched with a $200-\mu \mathrm{L}$ sterile 
pipette tip. Afterwards, the medium was displaced with fresh medium containing 2\% FBS. At 1 hour and 24 hours, the status of cell migration was observed using a microscope (Olympus, Japan) at $40 \times$ magnification by selecting 3 random fields of view. The images obtained were analyzed by Image $\mathrm{J}$ software, and the wound width (mean $\pm \mathrm{SD}$ ) was used to describe the migration ability of cancer cells.

\section{Transwell invasion assay}

Transwell invasion assay was conducted using Transwell chambers ( 24 wells, $8 \mu \mathrm{m}$ pore size). Briefly, $5 \times 10^{5}$ cells in $200 \mu \mathrm{L}$ serum-free medium were added to the upper chamber, while $600 \mu \mathrm{L}$ of culture medium with $10 \%$ FBS was loaded in the lower chamber. The chambers were incubated for 24 hours at $37^{\circ} \mathrm{C}$. The number of invaded cells on the lower side was counted in 5 random fields of view (200x) after fixation using paraformaldehyde.

\section{Lymphatic metastasis evaluation in the intraplantar model}

An intraplantar model of tumor was used to evaluate the lymphatic metastasis of MCTS1-overexpressed oral cancer cells. In detail, $5 \times 10^{5}$ SCC9 cells were subcutaneously implanted into the footpad region of the hind limb of $\mathrm{BALB} / \mathrm{c}$ Nude mice. After inoculation, the mice were fed for 30 days to allow for tumorigenesis. On the 30th day, the mice were sacrificed, and ipsilateral intraplantar lymph nodes (pLNs) and primary tumor tissues were sampled. The lymphatic metastasis and histomorphology of tumor were assessed by hematoxylin and eosin (HE) staining. The 4-6-week-old female BALB/c Nude mice were purchased from GemPharmatech (GemPharmatech, Jiangsu, China) with average body weight among 20-22 g. All mice were allocated to different experiment groups randomly and fed in specific pathogen free (SPF) environment. The animal experiments were approved by the Ethical Committee of the First Affiliated Hospital, Sun Yat-Sen University \{No. [2019] 069\}, and all procedures were performed in accordance with the institutional guidelines for the care and use of animals.

\section{Statistical analysis}

Data are expressed the mean $\pm \mathrm{SD}$, and statistical analyses were performed with SPSS v.20.0 software (IBM Corp, USA). Two-tail Student's $t$-test was used for comparing the mRNA, protein level, cell number, and the lymph node metastasis area, while chi-squared test was used to examine the difference in the lymph node metastasis incidence between the control and trial group. A $\mathrm{P}$ value $<0.05$ was regarded as a statistically significant difference.

\section{Results}

\section{MCTS1 expression in oral cancer}

Bioinformatic analyses of gene expression data in The Cancer Genome Atlas (TCGA) database revealed that MCTS1 was significantly highly expressed in head and neck cancer. The overall survival rate of head and neck cancer patients with high expression of MCTS1 was found to be markedly lower than that of patients with a low expression of MCTS1 (Figure 1A,B). Likewise, the disease-free survival rate was lower in the cases of head and neck cancer with high expression of MCTS1. This prompted us to further examine the MCTS1 activity in cancer development. Immunohistochemical scores indicated that high MCTS1 expression were present in the lymph node metastasispositive group (Figure 1C,D). The expression of MCTS1 in HOK and 4 kinds of oral cancer cells (SCC9, SCC15, SCC25, and Cal 27) was analyzed by western blotting and RT-PCR. As shown in Figure1E,F, the expression of MCTS1 in all oral cancer cell lines, except for SCC9, was highly expressed compared to normal HOKs. The large difference in MCTS1 level between normal and cancerous cells $(\mathrm{P}<0.01)$ indicates that elevated MCTS1 expression is related to the oncogenesis of oral cancer.

\section{MCTS1 facilitated tumor cell growth and proliferation}

MCTS1-overexpressed SCC9 and MCTS1-knockdown SCC25 were used to investigate the effect of MCTS1 on the cell growth and proliferation of oral cancer. As shown in Figure 2, SCC9 resulted in a high MCTS1 expression as reflected by the protein level determined by western blotting (Figure 2A), while SCC25 exhibited a relatively low expression level of MCTS1 due to knockdown by lentiviral shRNA (Figure 2E). Clearly, the upregulation of MCTS1 promoted the cell growth and proliferation (Figure 2B,C,D). However, knockdown of MCTS1 suppressed the growth and proliferation of oral cancer cells in terms of SCC9 (Figure 2F, G,H). The cell concentration and colony numbers in the SCC9 cell line were significantly greater than those in the vector group $(\mathrm{P}<0.01)$. These results indicate that the upregulation of MCTS1 in oral cancer cells can facilitate 

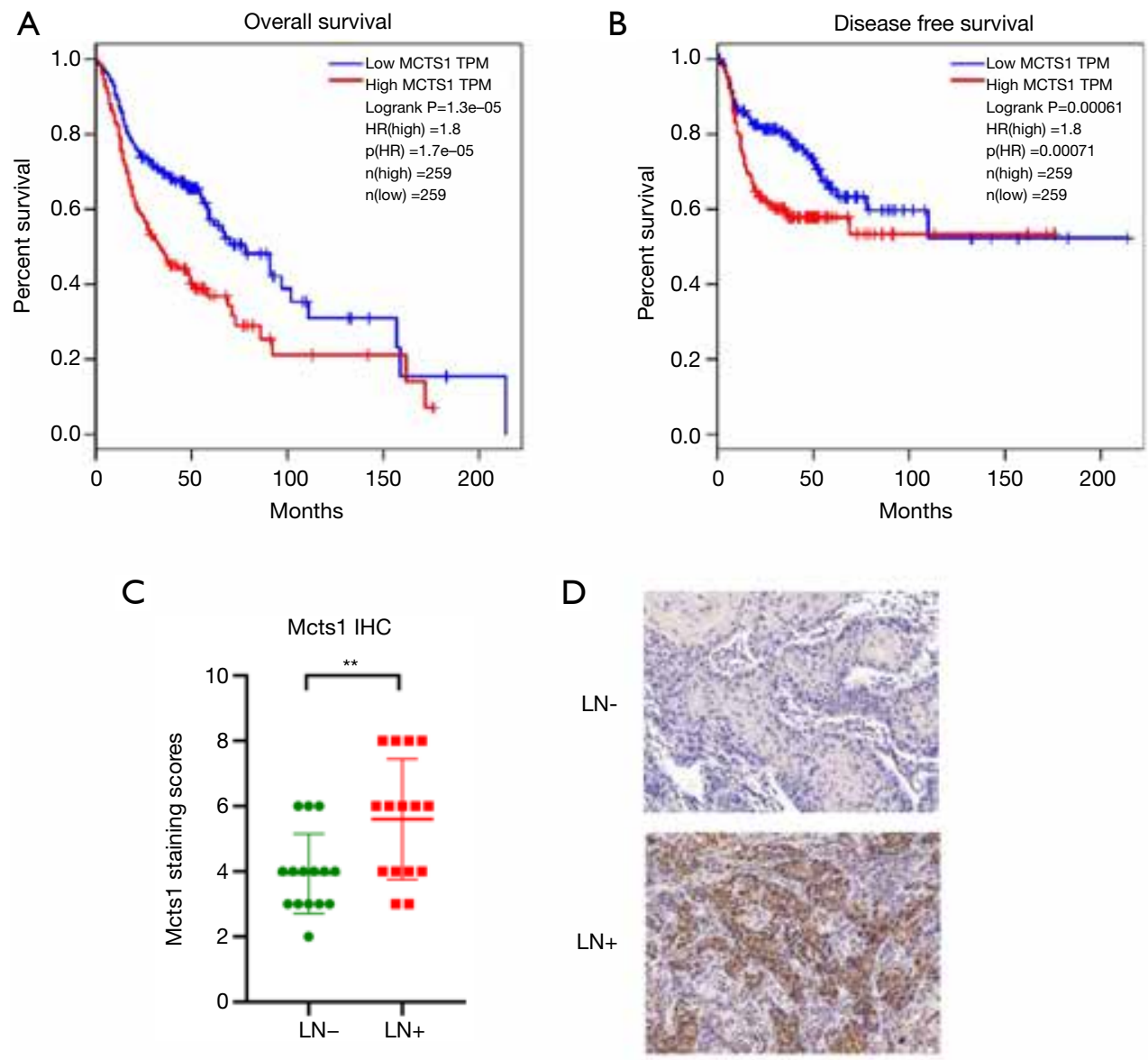

\section{$\mathrm{E}$}

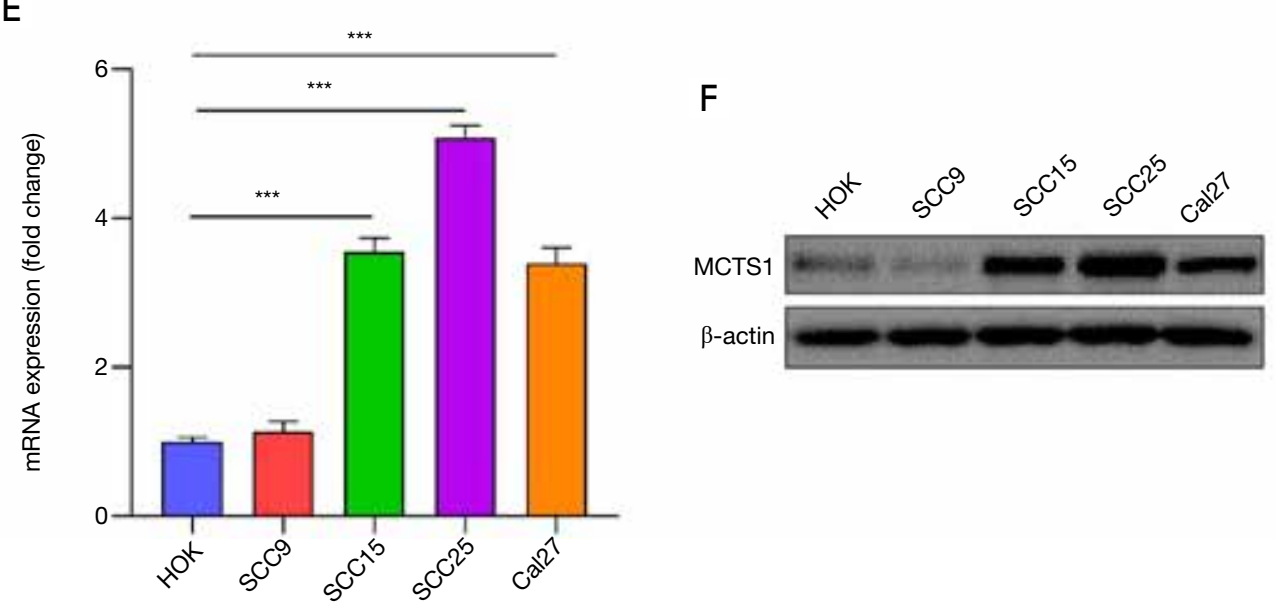

Figure 1 Survival analysis of MCTS1 in head and neck cancer using TCGA database. Overall survival rate (n=259, 50\% cutoff) (A) and disease-free survival rate ( $\mathrm{n}=259,50 \%$ cutoff) (B). Tissue microarray analysis of MCTS1 expression in patients with lymphatic node metastasis and nonmetastasis. MCTS1 scored by multiplying the proportion score and the intensity score using immunohistochemical staining (chi-squared test, $\left.{ }^{* *} \mathrm{P}<0.01\right)(\mathrm{C})$ and immunohistochemical images of lymphatic node metastasis (LN+) and nonmetastasis (LN-) (100× magnification) (D). Expression of MCTS1 in human oral keratinocytes (HOKs) and oral cancer cells (SCC9, SCC15, SCC25, and Cal27) quantified by RT-PCR (E) and western blotting (Two-tail Student's $t$-test, $\left.{ }^{* * *} \mathrm{P}<0.001\right)(\mathrm{F})$ of mRNA and MCTS1-associated protein, respectively. MCTS1, malignant T-cell-amplified sequence 1 . 
A

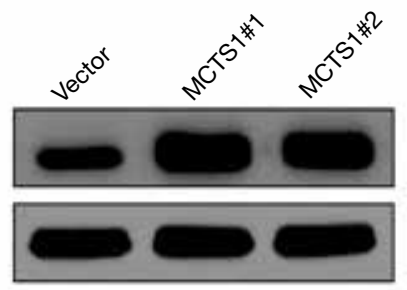

C

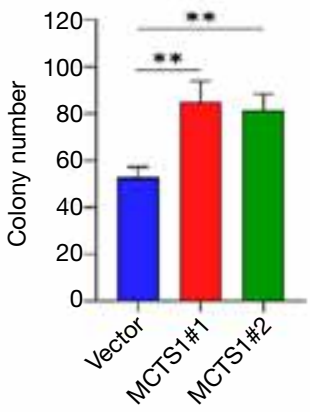

D
B

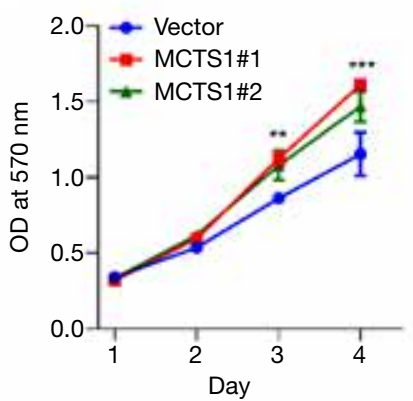

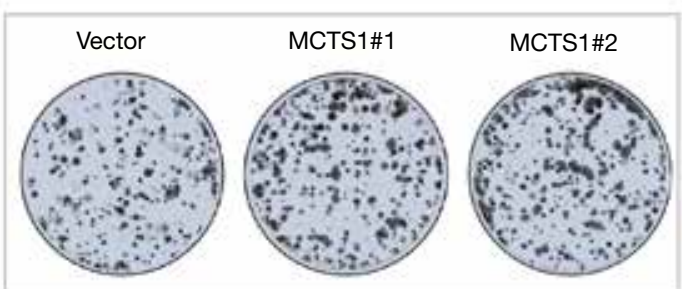

$\operatorname{SCC} 25$

E

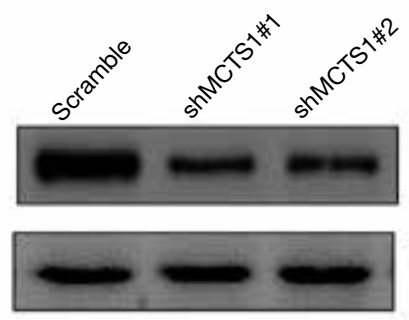

G

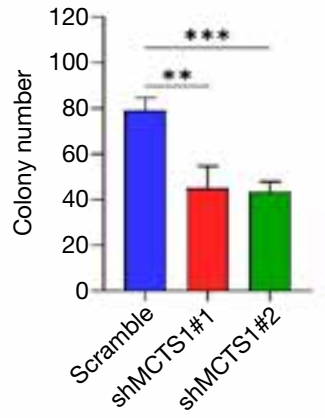

$\mathrm{H}$

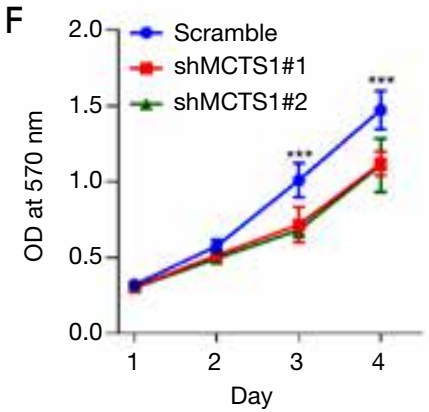

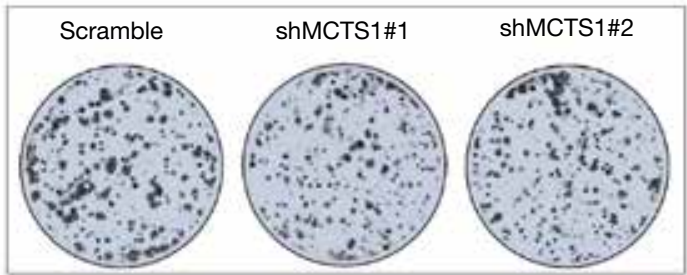

Figure 2 Effects of MCTS1 expression on tumor cell growth and proliferation (\#1 and \#2, two sets in parallel). (A) MCTS1-overexpressed stable transformant was validated by western blot assay in SCC9 cell lines. (B) Growth profiles of SCC25 cell lines according to MTT assay. (C) Colony formation histogram of SCC25 reflecting the growth ability of cells. (D) Representative colony formation images. (E) Lentiviral shRNA-mediated MCTS1 knockdown of the SCC25 cell line as detected by western blot assay. (F) Growth profiles of SCC9 cell lines tested by MTT assay. (G) Colony formation histogram of SCC9 reflecting the growth ability of cells. (H) Representative colony formation images. MCTS1, malignant T-cell-amplified sequence 1. Statistic analysis: Two-tail Student's $t$-test, ${ }^{* *} \mathrm{P}<0.01,{ }^{* * *} \mathrm{P}<0.001$, significantly different from vector or scramble group. 
cell growth and proliferation.

\section{MCTS1 promoted tumor cell migration and invasion}

Wound healing and Transwell invasion assays were performed to test the migration ability of MCTS1knockdown and MCTS1-overexpressed oral cancer cells. Compared to the vector group, SCC9 cells with high expression of MCTS1 exhibited enhanced wound healing and invasion abilities (Figure $3 A, B, C$ ). The cells were allowed to migrate for 24 hours, after which time it was observed that the scratches on the monolayers of MCTS1 overexpressed cell clone MCTS1\#1 and MCTS1\#2 had completely healed; meanwhile, the vector group was still in a weaker healing status. In contrast, the MCTS1 knockdown group of SCC25 cell clones shMCTS1\#1 and shMCTS1\#2 exhibited a marginal migration distance (Figure 3D). Likewise, the cell invasion events were relatively low, at less than half of the scramble (Figure $3 E, F$ ). These results demonstrate that knockdown of MCTS1 can suppress the migration and invasion abilities of oral cancer cells. Wound healing migration and invasion assays showed that promotional migration and invasion tropisms were induced by MCTS1 overexpression in oral cancers. This indicates that MCTS1 can potentially regulate cell proliferation and migration of oral cancer.

\section{Enhanced lymphatic metastasis mediated by MCTS1}

In an intraplantar oral cancer model, we found that high expression of MCTS1 significantly promoted lymphatic metastasis as revealed by the histological examination on lymph node (LN) metastasis incidence, as well as the area and weight of the tumor (Figure 4). Compared to the vector, the tumor showed enhanced invasion in the cases of MCTS1-overexpressed mice (paralel group \#1 and \#2), indicating that LN metastasis in the mice was more extensive (Figure 4A). Increased LN metastasis incidence was also present in the SCC25-bearing mice (Figure $4 B$ ), in which the percentage of $\mathrm{LN}$ metastasis was nearly 3 times greater. In addition, the $\mathrm{LN}$ metastasis area (Figure $4 C$ ) was significantly different between the vector and experimental groups (\#1 and \#2; $\mathrm{P}<0.05$ ). As for the tumor weight, there was no significant differences among the groups (Figure 4D). This can be reasonably explained by the concurrent tumor growth and the fact that lymphatic metastasis does not singly cause changes in tumor weight due to translocation. The enhanced $\mathrm{LN}$ metastasis incidence and area suggest that high expression of MCTS1 in malignant cells increases the probability of lymphatic metastasis of oral cancer.

\section{MCTS1 modified the EMT process in oral cancer cells}

To clarify the underlying mechanism of MCTS1facilitated metastasis, EMT-associated markers in MCTS1overexpressed SCC9 and MCTS1-knockdown SCC25 cells were determined by western blotting. Figure 5 shows the changes in E-cadherin, $\mathrm{N}$-cadherin, Vimentin, $\beta$-catenin, Snail, and Slug in human squamous carcinomas with different MCTS1 expression. Regarding the adhesion molecules, the E-cadherin level in MCTS1-overexpressed SCC9 cells decreased markedly while the level of $\mathrm{N}$-cadherin increased. Conversely, the levels of filament proteins increased in both cases of Vimentin and $\beta$-catenin. Transcription factor Slug was elevated in the SCC9 cell overexpressed MCTS1. However, in terms of SCC25, the EMT markers were reversely regulated by the knockdown of MCTS1. This indicates that MCTS1 plays an important role in the biological pathway of EMT.

\section{Discussion}

MCTS-1, an oncogene initially identified in human T-cell lymphoma, has been shown to induce cell proliferation and activate survival-related pathways (16). Diseases associated with MCTS1 include lymphoma and breast cancer. Gene ontology annotations related to this gene involve RNA binding and translation initiation factor activity $(17,18)$. According to the statistics from The Human Protein Atlas, ectopic expression of MCTS1 has been linked to several types of cancers, including head and neck cancer, renal cancer, and breast cancer, and its overexpression is an indicator for tumor cell proliferation, metastasis, and EMT (19). MCTS1 has been documented to be upregulated in oncogenesis that denatures cell cycle progression and fosters the growth of cancer cells $(20,21)$. However, the role of MCTS1 in regulating cell proliferation and migration of oral cancer is not well understood.

In the present study, we confirmed that MCTS1 was highly expressed in oral cancer compared to normal tissue. In vitro experiments provided evidence that knockdown of MCTS1 significantly receded the growth and proliferation abilities of oral cancer cells, while high expression of MCTS1 resulted in opposite oncogenesis, suggesting the oncogenic property of MCTS1 in oral cancer (22). The wound healing experiment revealed elevated expression 
A

scc9

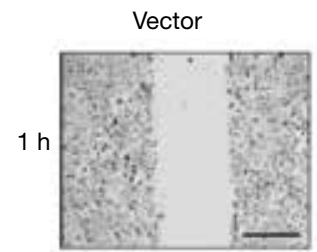

MCTS1\#1

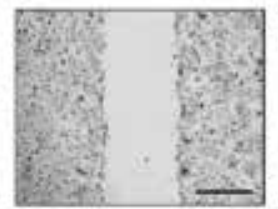

MCTS\#2
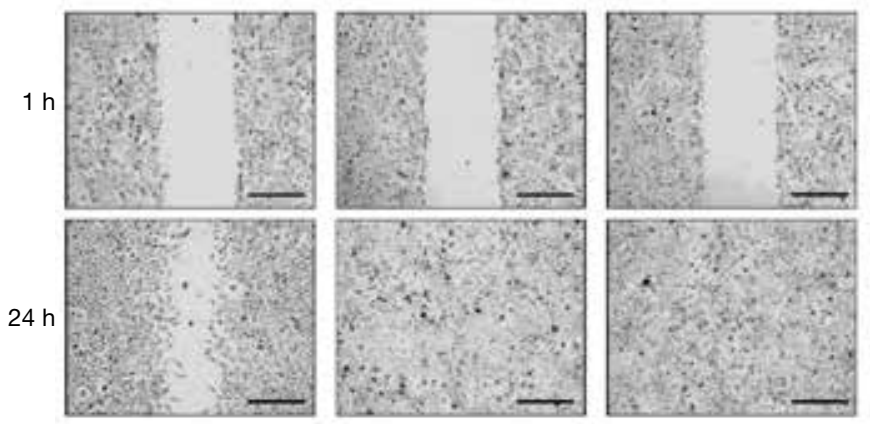

B

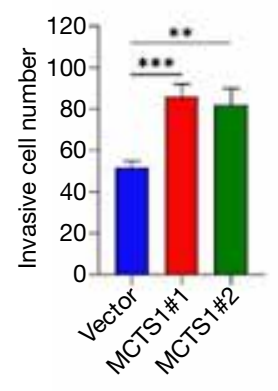

C
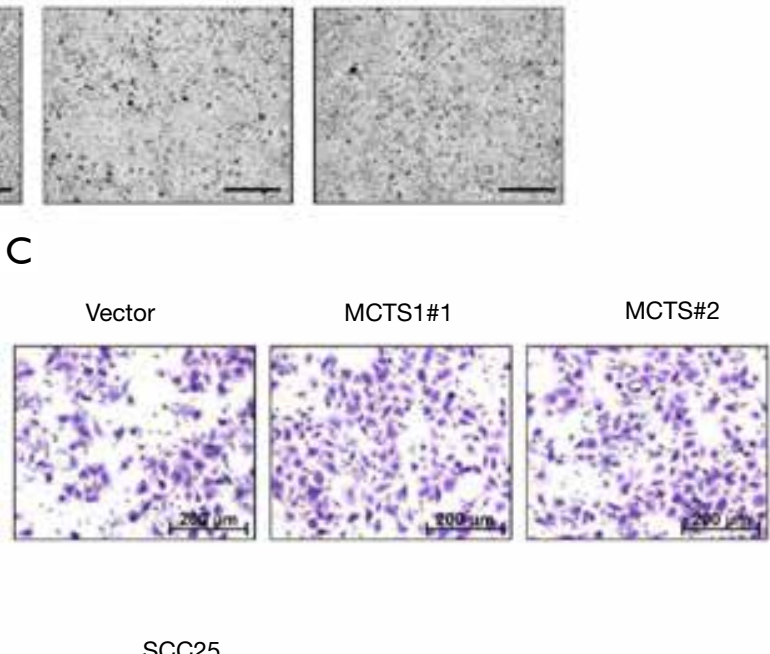

SCC25

D

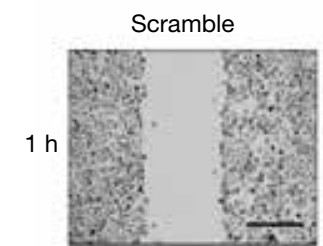

ShMCTS1\#1

ShMCTS\#2
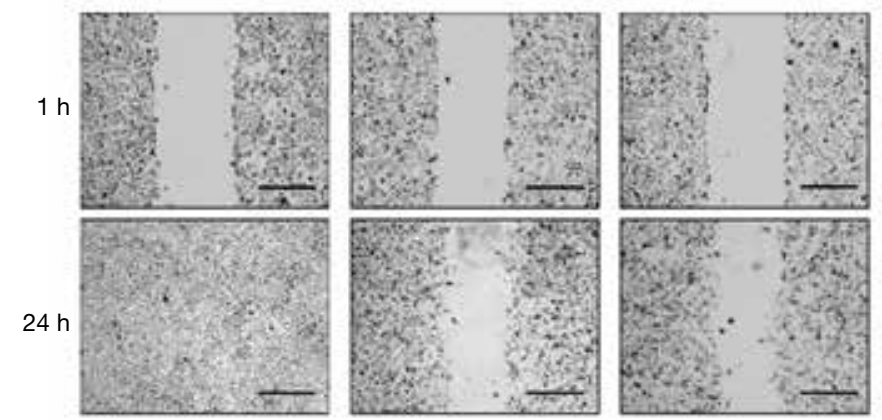

E

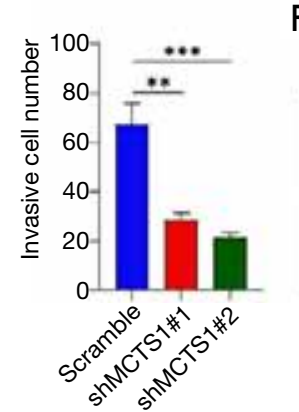

F

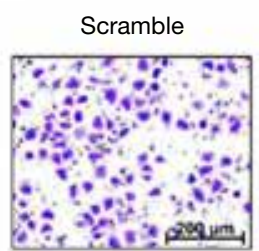

ShMCTS1\#1
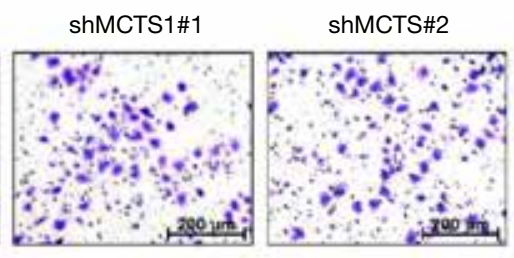

.

Figure 3 Cell migration and invasion assay of MCTS1-konckdown and overexpressed oral cancer cells evaluated by wound healing and Transwell invasion tests. (A) Wound healing of MCTS1-overexpressed SCC9 cells at 1 hour and 24 hours (scale bar =500 $\mu \mathrm{m}$ ). (B) Invasive cell number of MCTS1-overexpressed SCC9 across the Transwell membrane. (C) Representative migrated cells images for SCC9 cells. (D) Wound healing of MCTS1-knockdown SCC255 cells at 1 hour and 24 hours (scale bar $=500 \mu \mathrm{m}$ ). (E) Invasive cell number of MCTS1-knockdown SCC25 cells across the Transwell membrane. (F) Representative migrated cells images for the SCC25 line. MCTS1, malignant T-cell-amplified sequence 1. Statistic analysis: Two-tail Student's $t$-test, ${ }^{* *} \mathrm{P}<0.01,{ }^{* * *} \mathrm{P}<0.001$, significantly different from vector or scramble group. 
A

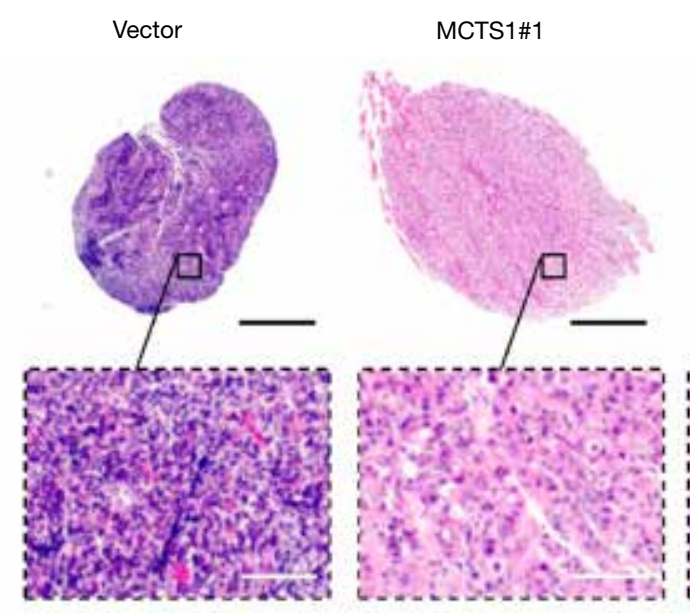

B

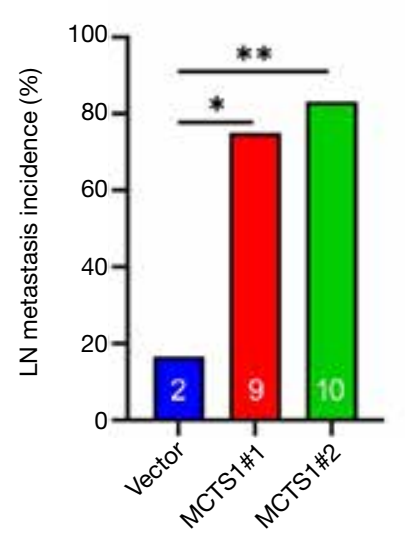

C

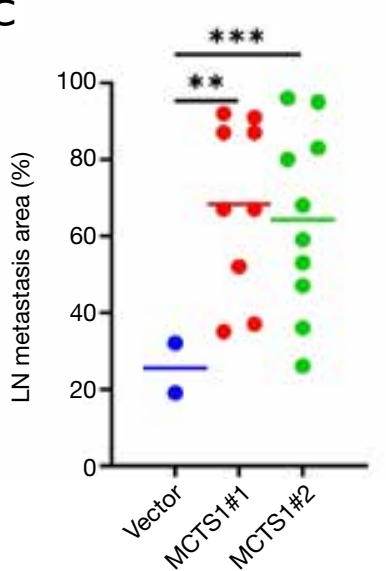

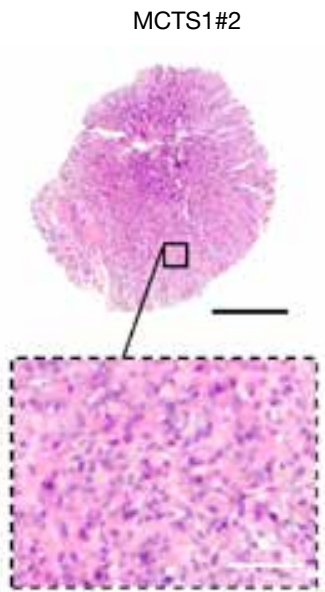

D

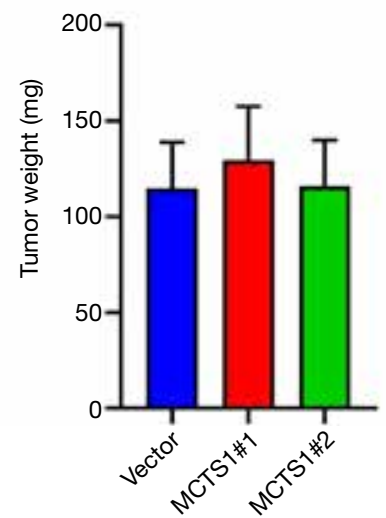

Figure 4 Lymphatic metastasis evaluation in an intraplantar model using nude mice. (A) Lymph node (LN) metastasis of vector group (n=11), MCTS1\#1 group ( $\mathrm{n}=12)$ and MCTS1\#2 group ( $\mathrm{n}=12)$ were characterized by hematoxylin and eosin (HE) staining (scale bar =200 $\mu \mathrm{m})$. (B) LN metastasis incidence of MCTS1 (\#1 and \#2) and vector groups in the histogram (according to chi-square test). (C) LN metastasis area in a scatter plot (according to 2-tailed Student's $t$-test). (D) Mean primary tumor weight among different groups. Statistic analysis: Two-tail Student's $t$-test, ${ }^{*} \mathrm{P}<0.05,{ }^{* *} \mathrm{P}<0.01,{ }^{* * *} \mathrm{P}<0.001$, significantly different from vector or scramble group.

of MCTS1 in oral cancer cells (SCC9) could substantially promote cell migration, whereas the migration ability of MCTS1-knockdown oral cancer cells (SCC25) was greatly limited. Findings from the Matrigel invasion assay were in line with the above observations. The invasive cell number in the SCC9 group was markedly higher than that in vector group. In contrast, the SCC25 cell line showed a significant increase in the invasive cell number in comparison to the scramble group. The results from wound healing and invasion assays firmly indicate that MCTS1 is involved in tumor growth and proliferation. The underlying mechanism for MCTS1 affecting cell multiplication has been speculated to be related to the inhibition of apoptosis, promotion of angiogenesis, enhancement of AKT activity, and protection of cells against programmed death under environmental stress (23).

The oncogenic properties of MCTS1 were verified by the in vitro experiments discussed above. In our in vivo experiment, the mice injected with MCTS1-overexpressed SCC9 cells developed more numerous and more severe lymphatic metastases. The results from the intraplantar model confirmed that MCTS1 could promote oral cancer metastasis in vivo, which accorded well with the in vitro results. This is the first study of its kind to demonstrate the 


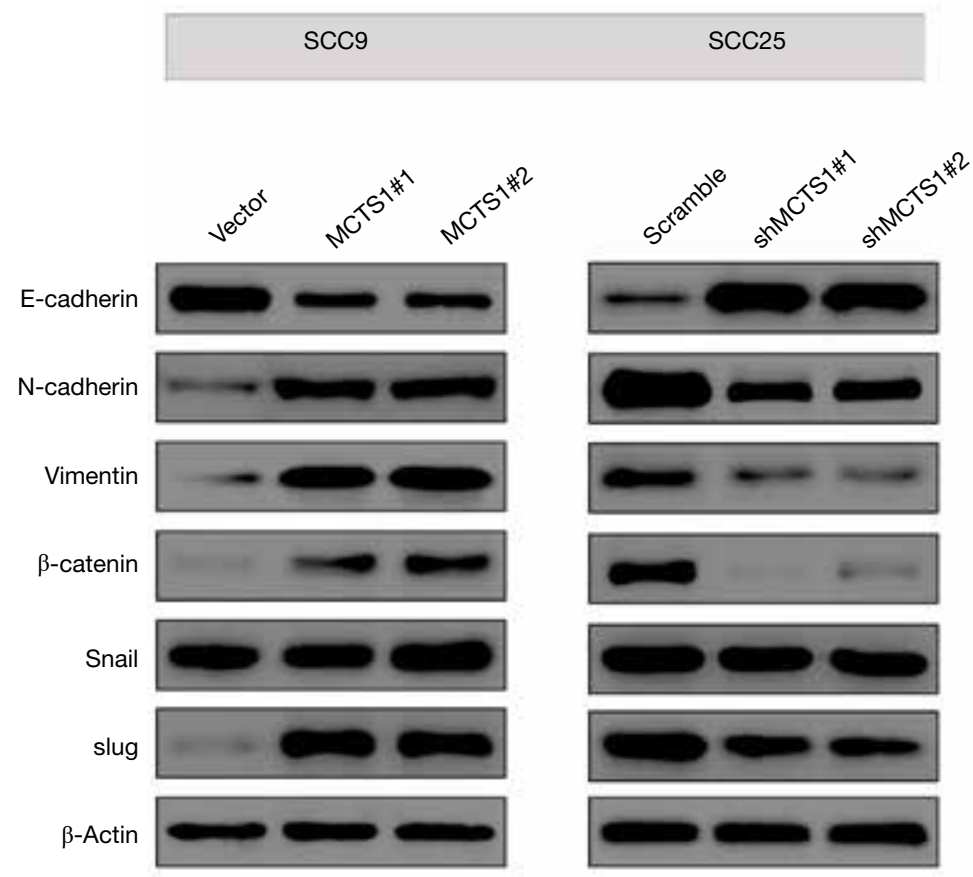

Figure 5 Changes in EMT markers affected by MCTS1 as analyzed by western blotting. E-cadherin, N-cadherin, Vimentin, $\beta$-catenin, Snail, and Slug were reversely regulated by MCTS1 in SCC9 with MCTS1 overexpression and SCC25 with MCTS1 knockdown. EMT, epithelial-mesenchymal transition; MCTS1, malignant T-cell-amplified sequence 1.

marked effect of MCTS1 on the metastasis of oral cancer cells. MCTS1, as biomarker, may thus be a potential target for the diagnosis and treatment of head and neck cancers.

Considering the vital role of EMT progress in tumor metastasis $(24,25)$, we examined the effects of MCTS1 on EMT in oral cancer cells. Results from western blotting showed that MCTS1 promoted EMT progress evinced by the fact that MCTS1 deficiency increased E-cadherin expression and decreased $\mathrm{N}$-cadherin, Vimentin, $\beta$-catenin, Snail, and Slug protein expression, while MCTS1 overexpression produced the opposite effects on the generation of EMT markers. The hallmark of EMT development is upregulation of $\mathrm{N}$-cadherin followed by downregulation of E-cadherin (26), in which a complex network of signaling pathways and transcription factors is involved. For example, EMT-inducing transcription factors, such as Snail and Slug, negatively regulate the expressions of E-cadherin and genes associated with mesenchymal phenotypes, resulting in the formation of migratory structure and the degradation of extracellular matrix $(27,28)$. In general, E-cadherin expression was negatively related to the expression of Vimentin, an intermediate filament protein, in the process of EMT $(29,30)$. The same events emerged in our study with oral cancer cells. In normal cells, $\beta$-catenin promotes the formation of adherens junction (AJ) by binding to E-cadherin, but it can induce EMT when released from the E-cadherin- $\beta$-catenin complex, showing a declined protein expression level (31). It follows that MTCS1 can modify the EMT process by regulating the adhesion molecules, transcription factors, and filament proteins. In our study, the EMT phenotype markers significantly changed in MCTS1-overexpressed oral cancer cells with upregulation of E-cadherin and downregulation of $\mathrm{N}$-cadherin, vimentin, $\beta$-catenin, Snail, and Slug. MCTS1 rendered oral cancer cells a mesenchymal property, featured by reducing cell-to-cell adhesion as regulated by E-cadherin and elevating the expressions of mesenchymal biomarkers. As reported by Weng et al., MCTS1 can promote EMT progression, cancer stemness, and M2 macrophage polarization in breast cancer through the MCT-1/miR-34a/ IL-6/IL-6R signaling axis (21).

\section{Conclusions}

MCTS1 overexpression is a hallmark of oncogenesis. In our study, we elucidated the biological significance of MCTS1 
in oral cancer evolution. MCTS1 can facilitate oral cancer cell growth and proliferation and promote tumor cell migration and invasion, thus enhancing the probability of oral cancer metastasis. The mechanism underlying the promotion of metastasis by MCTS1 was assumed to be associated with the ability of MCTS1 to modify the EMT process. Our findings provide a potential target for the diagnosis and therapy of oral cancer. However, how MCTS1 regulates EMT throughout the entire signaling cascade in oral cancer is still unknown and should be clarified by further research.

\section{Acknowledgments}

Funding: This work was supported by the National Nature Science Foundation of China (NSFC 81672659).

\section{Footnote}

Reporting Checklist: The authors have completed the ARRIVE reporting checklist. Available at http://dx.doi. org/10.21037/atm-21-2361

Data Sharing Statement: Available at http://dx.doi. org/10.21037/atm-21-2361

Conflicts of Interest: All authors have completed the ICMJE uniform disclosure form (available at http://dx.doi. org/10.21037/atm-21-2361). The authors have no conflicts of interest to declare.

Ethical Statement: The authors are accountable for all aspects of the work in ensuring that questions related to the accuracy or integrity of any part of the work are appropriately investigated and resolved. The study was conducted in accordance with the Declaration of Helsinki (as revised in 2013). All samples were obtained with informed consent and their use was approved by the Ethics Committee of the First Affiliated Hospital of Sun Yat-sen University (No. 2016074). The animal experiments were approved by the Ethical Committee of the First Affiliated Hospital, Sun Yat-Sen University (No. [2019] 069), and all procedures were performed in accordance with the institutional guidelines for the care and use of animals.

Open Access Statement: This is an Open Access article distributed in accordance with the Creative Commons Attribution-NonCommercial-NoDerivs 4.0 International
License (CC BY-NC-ND 4.0), which permits the noncommercial replication and distribution of the article with the strict proviso that no changes or edits are made and the original work is properly cited (including links to both the formal publication through the relevant DOI and the license). See: https://creativecommons.org/licenses/by-nc-nd/4.0/.

\section{References}

1. Hachem A, Nandi S. The oncogene mcts1. Transl Oncogenomics 2007;2:79-84.

2. Luch A. Cell cycle control and cell division: implications for chemically induced carcinogenesis. Chembiochem 2002;3:506-16.

3. Kontomanolis EN, Koutras A, Syllaios A, et al. Role of Oncogenes and Tumor-suppressor Genes in Carcinogenesis: A Review. Anticancer Res 2020;40:6009-15.

4. Casalino L, Verde P. Multifaceted Roles of DNA Methylation in Neoplastic Transformation, from Tumor Suppressors to EMT and Metastasis. Genes (Basel) 2020;11:922.

5. Prosniak M, Dierov J, Okami K, et al. A novel candidate oncogene, MCT-1, is involved in cell cycle progression. Cancer Res 1998;58:4233-7.

6. Herbert GB, Shi B, Gartenhaus RB. Expression and stabilization of the MCT-1 protein by DNA damaging agents. Oncogene 2001;20:6777-83.

7. Hsu HL, Choy CO, Kasiappan R, et al. MCT-1 oncogene downregulates $\mathrm{p} 53$ and destabilizes genome structure in the response to DNA double-strand damage. DNA Repair (Amst) 2007;6:1319-32.

8. Nandi S, Reinert LS, Hachem A, et al. Phosphorylation of MCT-1 by p44/42 MAPK is required for its stabilization in response to DNA damage. Oncogene 2007;26:2283-9.

9. Bohlen J, Harbrecht L, Blanco S, et al. DENR promotes translation reinitiation via ribosome recycling to drive expression of oncogenes including ATF4. Nat Commun 2020;11:4676.

10. Levenson AS, Thurn KE, Simons LA, et al. MCT-1 oncogene contributes to increased in vivo tumorigenicity of MCF7 cells by promotion of angiogenesis and inhibition of apoptosis. Cancer Res 2005;65:10651-6.

11. Wijetunga NA, Yu Y, Morris LG, et al. The head and neck cancer genome in the era of immunotherapy. Oral Oncol 2020;112:105040.

12. Shi B, Hsu HL, Evens AM, et al. Expression of the candidate MCT-1 oncogene in B- and T-cell lymphoid 
malignancies. Blood 2003;102:297-302.

13. Shi Y, Liao N, Zhang G, et al. Higher MCTS1 mRNA level in breast cancer may associate with an unfavorable outcome. Ann Oncol 2017;28:v74-108.

14. Tian C, Zeng S, Luo J. MCTS1 Directly Binds to TWF1 and Synergistically Modulate Cyclin D1 and C-Myc Translation in Luminal A/B Breast Cancer Cells. Onco Targets Ther 2020;13:5353-61.

15. Ma J, Zhu L, Zhou Z, et al. The calcium channel TRPV6 is a novel regulator of RANKL-induced osteoclastic differentiation and bone absorption activity through the IGF-PI3K-AKT pathway. Cell Prolif 2021;54:e12955.

16. Fleischer TC, Weaver CM, McAfee KJ, et al. Systematic identification and functional screens of uncharacterized proteins associated with eukaryotic ribosomal complexes. Genes Dev 2006;20:1294-307.

17. Reinert LS, Shi B, Nandi S, et al. MCT-1 protein interacts with the cap complex and modulates messenger RNA translational profiles. Cancer Res 2006;66:8994-9001.

18. Ahmed YL, Schleich S, Bohlen J, et al. DENR-MCTS1 heterodimerization and $t R N A$ recruitment are required for translation reinitiation. PLoS Biol 2018;16:e2005160.

19. Shih HJ, Chen HH, Chen YA, et al. Targeting MCT1 oncogene inhibits Shc pathway and xenograft tumorigenicity. Oncotarget 2012;3:1401-15.

20. Li Y, Wang B, Gui S, et al. Multiple Copies in T-Cell Malignancy 1 (MCT-1) Promotes the Stemness of NonSmall Cell Lung Cancer Cells via Activating Interleukin-6 (IL-6) Signaling through Suppressing MiR-34a Expression. Med Sci Monit 2019;25:10198-204.

21. Weng YS, Tseng HY, Chen YA, et al. MCT-1/miR-34a/ IL-6/IL-6R signaling axis promotes EMT progression, cancer stemness and M2 macrophage polarization in triple-negative breast cancer. Mol Cancer 2019;18:42.

22. Hsu HL, Shi B, Gartenhaus RB. The MCT-1 oncogene product impairs cell cycle checkpoint control and

Cite this article as: Huang $\mathrm{Z}, \mathrm{Su} \mathrm{Q}, \mathrm{Li}$ W, Ren H, Huang H, Wang A. MCTS1 promotes invasion and metastasis of oral cancer by modifying the EMT process. Ann Transl Med 2021;9(12):997. doi: 10.21037/atm-21-2361 transforms human mammary epithelial cells. Oncogene 2005;24:4956-64.

23. Jiang SL, Mo JL, Peng J, et al. Targeting translation regulators improves cancer therapy. Genomics 2021;113:1247-56.

24. Yeung KT, Yang J. Epithelial-mesenchymal transition in tumor metastasis. Mol Oncol 2017;11:28-39.

25. Pastushenko I, Blanpain C. EMT Transition States during Tumor Progression and Metastasis. Trends Cell Biol 2019;29:212-26.

26. Loh CY, Chai JY, Tang TF, et al. The E-Cadherin and N-Cadherin Switch in Epithelial-to-Mesenchymal Transition: Signaling, Therapeutic Implications, and Challenges. Cells 2019;8:1118.

27. Serrano-Gomez SJ, Maziveyi M, Alahari SK. Regulation of epithelial-mesenchymal transition through epigenetic and post-translational modifications. Mol Cancer 2016;15:18.

28. Wang Y, Shi J, Chai K, et al. The Role of Snail in EMT and Tumorigenesis. Curr Cancer Drug Targets 2013;13:963-72.

29. Satelli A, Li S. Vimentin in cancer and its potential as a molecular target for cancer therapy. Cell Mol Life Sci 2011;68:3033-46.

30. Richardson AM, Havel LS, Koyen AE, et al. Vimentin Is Required for Lung Adenocarcinoma Metastasis via Heterotypic Tumor Cell-Cancer-Associated Fibroblast Interactions during Collective Invasion. Clin Cancer Res 2018;24:420-32.

31. Chaw SY, Abdul Majeed A, Dalley AJ, et al. Epithelial to mesenchymal transition (EMT) biomarkers--E-cadherin, beta-catenin, APC and Vimentin--in oral squamous cell carcinogenesis and transformation. Oral Oncol 2012;48:997-1006.

(English Language Editor: J. Gray) 